(C) 2017 Universidad Nacional Autónoma de México, Facultad de Estudios Superiores Zaragoza.

Este es un artículo Open Access bajo la licencia CC BY-NC-ND (http://creativecommons.org/licenses/by-nc-nd/4.0/).

TIP Revista Especializada en Ciencias Químico-Biológicas, 20(2): 48-52, 2017.

DOI: 10.1016/j.recqb.2017.04.005

\title{
Casos de blastomicosis reportados en México
}

\author{
Elva Bazán-Mora, Érika Córdova-Martínez, Rubén López-Martínez, \\ Gabriela Morales-Flores, Sandra B. Villegas-García, \\ Orlanda A. Guzmán-Santos y Laura R. Castañón-Olivares \\ Unidad de Micología, Facultad de Medicina, Universidad Nacional Autónoma de México, \\ Avenida Universidad \# 3000, Col. Ciudad Universitaria, Deleg. Coyoacán, C.P. 04510 \\ Ciudad de México, México E-mail: 1rcastao@unam.mx
}

\begin{abstract}
Resumen
Aunque la blastomicosis es una enfermedad que afecta a mamíferos y se considera endémica de América del Norte, también se han reportado casos autóctonos en otros lugares del mundo. En el presente trabajo, se efectuó un análisis cualitativo de los casos humanos de blastomicosis diagnosticados en México, iniciando con una búsqueda bibliográfica electrónica del período comprendido de 1898 a 2016 y para ayudar al estudio de los datos de cada paciente, se elaboró un cuadro comparativo. Se encontraron seis casos de blastomicosis durante el período examinado. Los pacientes fueron del género masculino, distribuídos en diferentes etapas de la vida y el antecedente de haber residido o visitado zonas consideradas endémicas de la enfermedad en Estados Unidos de América (EUA), por lo que se concluye que los casos mexicanos de blastomicosis publicados, no son autóctonos.
\end{abstract}

Palabras Clave: blastomicosis, Blastomyces dermatitidis, infección pulmonar, micosis sistémica, México

\section{Blastomycosis cases reported in México}

\begin{abstract}
Although blastomycosis is a mammal's disease, endemic in North America, indigenous cases have been reported elsewhere in the world. The present report presents a qualitative analysis of human cases of blastomycosis diagnosed in Mexico, starting with an electronic bibliographic search which covered the period from 1898 to 2016. We made a data collection of each patient. Six cases of blastomycosis were found during such period.

All patients were male and distributed in different stages of life, and had resided in areas in the United States (USA) were the disease is endemic, which leads to conclude that published Mexican cases of blastomycosis, are not autochthonous.
\end{abstract}

Key Words: blastomycosis, Blastomyces dermatitidis, lung infection, systemic fungal infection, México.

Nota: Artículo recibido el 27 de septiembre de 2016 y aceptado el 26 de abril de 2017. 


\section{INTRODUCCIÓN}

B lastomyces dermatitidis es el nombre del hongo anamórfico, causante de la infección en mamíferos denominada blastomicosis. La fase sexual de este hongo heterotálico se conoce como Ajellomyces dermatitidis; es también un organismo dimórfico cuyo crecimiento micelial da lugar a conidios redondos sésiles o pedunculados y la forma parasitaria en el huésped mamífero, se presenta como levaduras redondas unigemantes, con blastoconidios de base ancha (Castillo et al., 2016; Saccente \& Woods, 2010; Klein \& Tebbets, 2007; Li et al., 2013; López-Martínez \& Méndez-Tovar, 2012). La blastomicosis puede ser adquirida por vía aérea (inhalación) o por vía cutánea (inoculación en la piel). La vía aérea es la forma más común de contagio, por lo que el aparato respiratorio es el más afectado; a partir de aquí y dependiendo del estado inmune del huésped, el hongo puede alcanzar la vía hematógena y a través de ella viajar a cualquier otro tejido por lo que su diseminación en piel y huesos, se presenta con cierta frecuencia y desafortunadamente se asocia a un mal pronóstico. Por el contrario, las infecciones cutáneas primarias, generalmente son benignas y no atentan contra la vida de los pacientes (López-Martínez \& Méndez-Tovar, 2012; Davies et al., 2013).

Son pocos los estudios ambientales que se han efectuado, pero relatan que la forma micelial infectante del hongo, se aisla de suelos húmedos, ricos en materia orgánica, sombreados y con $\mathrm{pH}<6$, condiciones que se presentan en los márgenes de ríos, lagos, riveras y zonas lacustres con árboles caídos (Castillo et al., 2016). La blastomicosis, reportada principalmente en humanos, cánidos y felinos es una infección considerada como endémica del noreste de los Estados Unidos de América (Wisconsin tiene registrada la mayor incidencia) y el sureste de Canadá (siendo Ontario el estado con mayor número de pacientes reconocidos); sin embargo, aunque con poca frecuencia, existen reportes fuera de esas áreas, en el mismo EUA e inclusive en otros países como Sudáfrica, Zimbabwe e India, son lugares donde aparentemente los casos diagnosticados se han referido como autóctonos (Castillo et al., 2016; López-Matínez \& Méndez-Tovar, 2012; Davies et al., 2013; Pfaff et al., 2014).

La presente nota científica, hace una descripción de los casos de blastomicosis diagnosticados en México, con el fin de extraer algún significado que permita deducir la situación epidemiológica a partir de lo hasta ahora publicado sobre el tema.

\section{Materiales y métodos}

Se hizo una búsqueda bibliográfica exhaustiva en el período 1898-2016 para encontrar y analizar los casos mexicanos de blastomicosis registrados. Las fuentes de información fueron las bases de datos incluidas en Imbiomed, Latindex, Lilacs,
Publindex, PubMed, que incluyen revistas especializadas en micología, microbiología, especialidades médicas (neumología, infectología y oncología entre otras). La búsqueda se efectuó con las palabras clave: blastomicosis, enfermedad de Gilchrist, Blastomyces dermatitidis y México. Una vez localizada la literatura, se elaboró un cuadro comparativo con algunos datos clínico-epidemiológicos de los casos encontrados.

\section{Resultados}

La revisión bibliográfica señaló seis artículos (MartínezBáez et al., 1954; Velázquez et al., 2003; Rodríguez-Mena et al., 2010; García Morúa et al., 2010; Salas-Alanís et al., 2013; Bonifaz et al., 2016) en donde coincidieron cuatro características: pacientes mexicanos, con diagnóstico de blastomicosis, diagnosticados en México y con antecedentes de haber habitado o visitado zonas consideradas endémicas de blastomicosis, en EUA; esta última característica, es vista como el principal factor epidemiológico de riesgo, para contraer la infección. Se muestran en el Cuadro I, algunos datos de los pacientes investigados; el género, origen y ocupación que epidemiológicamente no son considerados factores de riesgo.

A pesar de que la infección está reportada en ambos géneros, no se presentó ningún caso del género femenino y la edad varió desde 3 hasta 70 años, pero de los seis pacientes, cuatro se distribuyeron entre la $4^{\mathrm{a}}$. y $6^{\mathrm{a}}$. década de la vida (Figura 1).

Aunque los casos son abordados por los autores de diferentes maneras, se observó que en la mayoría, la historia de la enfermedad siguió el curso predecible, infección pulmonar diseminada a otros tejidos (Martínez-Báez et al., 1954; Velázquez et al., 2003; García Morúa et al., 2010; SalasAlanís et al., 2013). Aparentemente hay reporte de dos casos de blastomicosis cutánea primaria, uno quedó bien establecido (Rodríguez-Mena et al., 2010), pero el otro en el que las lesiones se asocian con picadura de insecto, no se menciona si se investigaron antecedentes de infección pulmonar previa (Bonifaz et al., 2016) (Figura 2). Debido al tipo de blastomicosis, la situación inmunológica de los pacientes, diagnóstico oportuno y el tratamiento instaurado, cuatro pacientes (Velázquez et al., 2003; Rodríguez-Mena et al., 2010; Salas-Alanís et al., 2013; Bonifaz et al., 2016) se recuperaron y sólo dos (Martínez-Báez et al., 1954; García Morúa et al., 2010) murieron. Llama la atención que en los casos sistémicos, se mencionan signos y síntomas que involucran varios tejidos; sin embargo, no se dice nada sobre la observación ni el aislamiento del hongo de los diversos especímenes patológicos revisados.

La descripción del diagnóstico microbiológico, no es completo en todos los casos. A excepción de un paciente (García Morúa et al., 2010), todos los demás reportan la observación en 


\begin{tabular}{|c|c|c|c|c|c|}
\hline \multirow{2}{*}{$\begin{array}{c}\text { Autores y año } \\
\text { de publicación }\end{array}$} & \multicolumn{4}{|c|}{ Datos epidemiológicos* } & \multirow{2}{*}{ Tipo de blastomicosis } \\
\hline & Residencia en EUA & Género & Origen & Ocupación & \\
\hline $\begin{array}{c}\text { Martínez-Báez et al., } \\
1954\end{array}$ & California & Masculino & Guanajuato & Campesino & $\begin{array}{l}\text { Sistémica y diseminada (pulmonar, } \\
\text { ósea y cutánea) }\end{array}$ \\
\hline Velázquez et al., 2003 & California e Illinois & Masculino & Guerrero & Preescolar & $\begin{array}{l}\text { Sistémica y diseminada (pulmonar y } \\
\text { cutánea) }\end{array}$ \\
\hline $\begin{array}{l}\text { García Morúa et al., } \\
2010\end{array}$ & No especificado & Masculino & No referido & No referida & Sistémica (prostática) \\
\hline $\begin{array}{l}\text { Rodríguez-Mena et } \\
\text { al., } 2010\end{array}$ & Illinois & Masculino & Jalisco & Albañil & Cutánea primaria \\
\hline $\begin{array}{l}\text { Salas-Alanís et al., } \\
2013\end{array}$ & Texas & Masculino & Nuevo León & Chofer & $\begin{array}{l}\text { Sistémica y diseminada (pulmonar, } \\
\text { prostática ósea y cutánea) }\end{array}$ \\
\hline Bonifaz et al., 2016 & $\begin{array}{l}\text { Arizona, Wisconsin, } \\
\text { Illinois, Ohio e } \\
\text { Indiana }\end{array}$ & Masculino & No referido & Campesino & Cutánea ¿primaria? \\
\hline
\end{tabular}

Cuadro I. Datos epidemiológicos de seis pacientes mexicanos diagnosticados con blastomicosis.

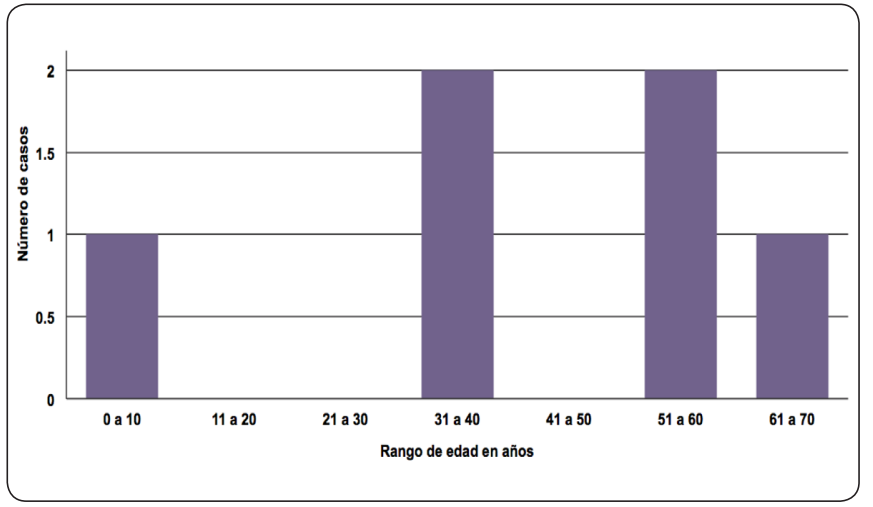

Figura 1. Distribución por edad de los casos de blastomicosis diagnosticados en México.

examen directo con $\mathrm{KOH}$ de levaduras unigemantes con blastoconidios de base ancha unidos a la levadura madre y el aislamiento del hongo en cultivo a $25^{\circ} \mathrm{C}$. De cinco autores, uno no hace la descripción del cultivo obtenido (Salas-Alanís et al., 2013), ni muestra fotos que documenten su hallazgo. Sólo en un caso (Velázquez et al., 2003) se menciona el uso de pruebas serológicas y en dos casos (Velázquez et al., 2003; Bonifaz et al., 2016) la identidad del cultivo aislado fue corroborada por análisis del DNA.

Finalmente llama la atención que únicamente en un caso se efectuaron pruebas de sensibilidad antifúngica al cultivo obtenido (Bonifaz et al., 2016); sin embargo, en el texto nunca se menciona en qué condiciones se llevaron a cabo las pruebas, ya que $B$. dermatitidis es considerado un organismo de riesgo biológico nivel 3 , por lo que las condiciones de incubación de la forma micelial (fase infectante del hongo), deben manejarse bajo estricto control de bioseguridad.

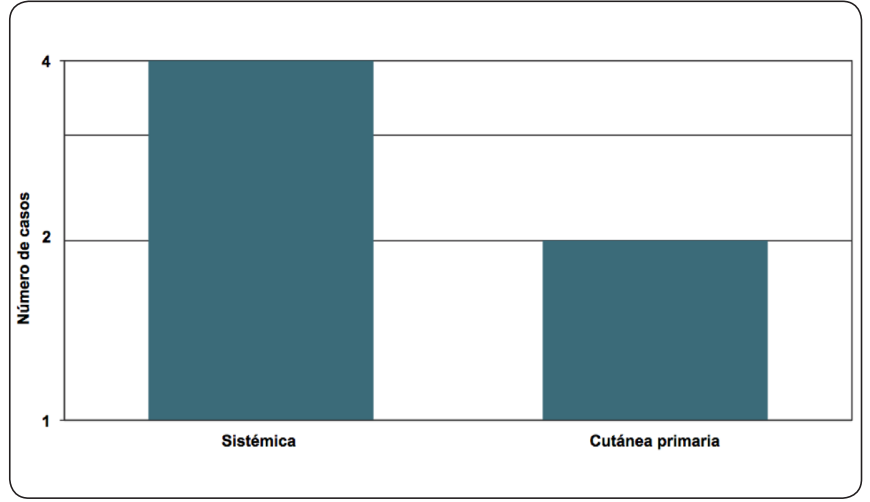

Figura 2. Formas clínicas de los casos de blastomicosis diagnosticados en México.

\section{Discusión}

Los casos analizados exponen que la blastomicosis en pacientes mexicanos sigue el mismo patrón reportado, para los pacientes aquejados por esta micosis en EUA como: predominio en el género masculino y en las etapas de la vida de mayor actividad, presentaciones benignas en baja frecuencia (casos cutáneos primarios) y casos sistémicos con mayor incidencia. El índice de mortalidad referido a la blastomicosis, depende en gran parte de la situación inmunológica del paciente, aun cuando el tratamiento se haya establecido.

Respecto a los estudios micológicos, esta revisión hace evidente que debe hacerse un esfuerzo por efectuar el examen directo y el cultivo del hongo; las imágenes de cortes histológicos en algunos artículos, no muestran claramente las formas parasitarias del agente; hay que recordar que el hallazgo en el tejido de cúmulos de levaduras unigemantes no es característico de $B$. dermatitidis. 
Es notable que a partir del primer caso mexicano de blastomicosis reportado en la literatura, pasaron 49 años para que se publicara el siguiente; sin embargo, el periodo posterior en que los subsiguientes casos han sido publicados es mucho menor, lo que refleja dos cosas: 1) ha aumentado el número de personas infectadas en EUA que regresan a México y 2) el personal médico que atiende a los pacientes, toma en cuenta el diagnóstico diferencial a la blastomicosis y en consecuencia efectúa los análisis de laboratorio pertinentes para la confirmación diagnóstica.

Una especie endémica es aquella que se distribuye en un ámbito geográfico reducido y que no se encuentra de forma natural en otras partes del mundo (Hennigan, 2013), bajo esa definición, los casos de blastomicosis diagnosticados en países de África, Israel e India, además de esporádicos, no han sido aclarados como realmente autóctonos.

Un factor epidemiológico determinante para la adquisición de la enfermedad es la exposición del humano al hongo. En EUA el aislamiento del hongo a partir de la naturaleza ha sido muy difícil, los estudios concluyen que muy probablemente el nicho ambiental para $B$. dermatitidis es el suelo con vegetación en descomposición, especialmente en la proximidad de lagos y ríos que forman la cuenca $\left(2,200 \mathrm{Km}^{2}\right)$ del río Mississippi (7,750 Km de recorrido) (Castillo et al., 2016), descripción muy amplia que no define los parámetros específicos que hacen que B. dermatitidis sea una especie propia y exclusiva de esa área.

México, cumple con la característica de poseer lagos y ríos en cuyos márgenes se localizan materiales vegetales en descomposición; sin embargo, al no conocer las condiciones ecológicas concretas del ecosistema y especialmente aquellas del micro-hábitat que permiten el crecimiento de $B$. dermatitidis, no podemos afirmar que en nuestro país, existan los escenarios ambientales adecuados para la presencia de $B$. dematitidis y por consecuencia, la fuente de infección para el humano.

\section{Conclusiones}

En México, no hay casos publicados de blastomicosis autóctonos, pero su hallazgo demuestra que hay personal capacitado para efectuar el diagnóstico; sin embargo, llama la atención que hasta la fecha, en un periodo de 63 años, se han reportado solamente seis casos en México, a pesar de la constante migración de mexicanos hacia el país vecino, dato que probablemente indica un subregistro de la enfermedad. Es importante destacar la necesidad de un constante adiestramiento del personal médico, para poder hacer diagnósticos diferenciales que incluyan a los hongos, en especial en casos de pacientes con antecedentes de migración hacia EUA, en quienes ante una sospecha de infección iniciada en vías respiratorias, se debe descartar, entre otras, a micosis sistémicas como la coccidioidomicosis y la blastomicosis. La continua migración de nuestros compatriotas a EUA, en busca de una mejor calidad de vida y el regreso a nuestro país de muchos de ellos, aumenta las posibilidades de infección con agentes no reconocidos en México, por lo que el diseño de un proceso sistemático, ordenado y planificado de observación, medición y registro de los casos-enfermedad, debería ser instaurado, con el argumento de que los incidentes de blastomicosis, aunque raros, se están presentando en nuestro país.

La presente nota científica, fue una investigación de tipo descriptivo en donde la aportación al conocimiento científico fue explicar, mediante la referencia de características epidemiológicas, la ausencia de casos autóctonos de blastomicosis en México, ya que en algún momento la literatura médica-micológica mexicana, ha dejado ver la posibilidad de que exista endemismo, apoyados en que prácticamente, por las múltiples condiciones ambientales prevalentes en nuestro medio, todas las micosis superficiales y sistémicas reportadas en el mundo, existen en México, conclusión que al menos para la blastomicosis, es inaceptable, hasta no demostrar que la ecología específica que soporta la sobrevivencia y desarrollo de $B$. dermatitidis se presenta en México.

\section{Referencias}

Bonifaz, A., Morales, D., Morales, N., Mercadillo, P., González, G.M., Hernández-Hernández, F., Araiza, J. \& Vázquez-González, D. (2016). Cutaneous blastomycosis. An imported case with good response to itraconazole. Rev. Iberoam. Micol. 33, 51-54 http:// dx.doi.org/10.1016/j.riam.2015.05.001

Castillo, C.G., Kauffman, C.A. \& Miceli, M.H. (2016). Blastomycosis Infect. Dis. Clin. N. Am. 30, 247-264 http://dx.doi.org/10.1016/j. idc.2015.10.002.

Davies, J.L., Epp, T. \& Burgess, H.J. (2013). Prevalence and geographic distribution of canine and feline blastomycosis in the Canadian prairies. Can. Vet. J. 54, 753-760.

García Morúa, A., Gutiérrez García, J.D., Valdés Sepúlveda, F., Lozano Salinas, J.F. \& Gómez Guerra, L.S. (2010). Blastomicosis prostática: presentación de un caso y revisión de la literatura. Actas Urol. Esp. 34, 206-217.

Hennigan, T. The ecology book (Master Books, Nueva York, 2013).

Klein, B.S. \& Tebbets, B. (2007). Dimorphism and virulence in fungi. Curr. Opin. Microbiol. 10, 314-319.

Li, W., Sullivan, T.D., Walton, E., Averette, A.F., Sakthikumar, S., Cuomo, C.A., Klein, B.S. \& Heitman, J. (2013). Identification of the mating-type (MAT) locus that controls sexual reproduction of Blastomyces dermatitidis. Eukaryot Cell 12, 109-117 DOI: 10.1128/EC.00249-12.

López-Martínez, R. \& Méndez-Tovar, L.J. (2012). Blastomycosis. Clin. Dermatol. 30, 565-572. doi.org/10.1016/j. clindermatol.2012.01.002.

Martínez-Báez, M., Reyes-Mota, A. \& González-Ochoa, A. (1954). Blastomicosis norteamericana en México. Rev. Inst. Salub. Enfem. Trop. 14, 225-232.

Pfaff, B.L., Agger, W.A. \& Volk, T.J. (2014). Blastomycosis diagnosed in a nonhyperendemic area. WMJ 113, 11-20.

Rodríguez-Mena, A., Mayorga, J., Solís-Ledesma, G. \& BarbaGómez, J. (2010). Blastomicosis: presentación de un caso importado en México, con lesiones exclusivamente cutáneas. Rev. Iberoam. Micol. 27, 210-212 http://dx.doi:10.1016/j. 
riam.2010.05.001.

Saccente, M. \& Woods, G.L. (2010). Clinical and laboratory update on blastomycosis. Clin. Microbiol. Rev. 23, 367-381 DOI:10.1128/ CMR.00056-09.

Salas-Alanís, J.C., Martínez, M.F., García-Meléndez, M., González, B.L. \& Ocampo-Candiani, J. (2013). Blastomycosis imported to
Monterrey, México: fifth case reported in México. Mycoses 56, 495-497 DOI:10.1111/myc.12051.

Velázquez, R., Muñoz-Hernández, B., Arenas, R., Taylor, M.L., Hernández-Hernández, F., Manjarrez, M.E. \& López-Martínez, R. (2003).An imported case of Blastomyces dermatitidis infection in Mexico. Mycopathologia 156, 263-267. 